\title{
Spectroscopy including XYZ states at BESIII
}

\author{
Liangliang Wang ${ }^{* \dagger}$ \\ Institute of High Energy Physics, Beijing, China \\ E-mail: Llwangdihep.ac.cn
}

\begin{abstract}
With the unique $e^{+} e^{-}$data samples at energies between 3.8 and $4.6 \mathrm{GeV}$, significant contributions on the charmonium and charmonium-like states (i.e. XYZ states) are made by BESIII. We review the recent studies of the XYZ states at BESIII. These results include the observations of $e^{+} e^{-} \rightarrow$ $\gamma \mathrm{X}(3872)$ and $e^{+} e^{-} \rightarrow \pi^{+} \pi^{-} \mathrm{X}(3823)$, the discovery of the $Z_{c}$ family and the measurements of the hadron plus charmonium channels in $e^{+} e^{-}$annihilation which provide oppotunities for the investigation of the vector exotic $\mathrm{Y}$ states and connections between these XYZ states.
\end{abstract}

16th International Conference on B-Physics at Frontier Machines

2-6 May 2016

Marseille, France

*Speaker.

${ }^{\dagger}$ For BESIII Collaboration 


\section{Introduction}

Numerous new charmonium and charmonium-like states were observed at B-factories in the last decades [四]. Some of them are good charmonium candidates, while many of them are not. Many interpretations are proposed for the nature of these exotic states [ [ $]$ ], i.e. XYZ states. But there are still many open questions which need further studies, both experimentally and theoretically.

The BESIII [B] experiment at BEPCII is designed to collect $e^{+} e^{-}$collision data in the $\tau$ charm energy region. BESIII has also collected data samples for XYZ studies at higher energy points, especially around the known vector charmonium or charmonium-like states like $\psi(4040)$, $\mathrm{Y}(4260), \mathrm{Y}(4360), \psi(4415)$ and $\mathrm{Y}(4660)$ [ [1], 6 ]. A large data sample at $4.18 \mathrm{GeV}$ which is near $\psi(4170)$ was taken in 2016.

\section{Studies of $X$ states at BESIII}

\subsection{Observation of $e^{+} e^{-} \rightarrow \gamma \mathrm{X}(3872)$}

With the data samples at $4.009 \mathrm{GeV}, 4.230 \mathrm{GeV}, 4.260 \mathrm{GeV}$ and $4.360 \mathrm{GeV}$, BESIII observed for the first time the process $e^{+} e^{-} \rightarrow \gamma \mathrm{X}(3872)$ with $\mathrm{X}(3872) \rightarrow \pi^{+} \pi^{-} J / \psi$ [四]. The invariant mass of $\pi^{+} \pi^{-} J / \psi$ for $\mathrm{X}(3872)$ candidates is shown in Fig. $\mathrm{W}$ (left). The statistical significance of $\mathrm{X}(3872)$ is $6.3 \sigma$ and the measured mass is $(3871.9 \pm 0.7 \pm 0.2) \mathrm{MeV} / c^{2}$ where the first uncertainty is statistical and the second systematic. A width of $\left(0.0_{-0.0}^{+1.7}\right) \mathrm{MeV}$ or less than $2.4 \mathrm{MeV}$ at the $90 \%$ C.L. was obtained by a fit with a floating width. These results are consistent with previous measurements [ [8]. The measured Born cross section $\sigma^{B}\left(e^{+} e^{-} \rightarrow \gamma X(3872) \rightarrow \gamma \pi^{+} \pi^{-} J / \psi\right)$ is shown in Fig. W (right) which supports strongly the existence of the radiative transition process $\mathrm{Y}(4260) \rightarrow \gamma \mathrm{X}(3872)$.
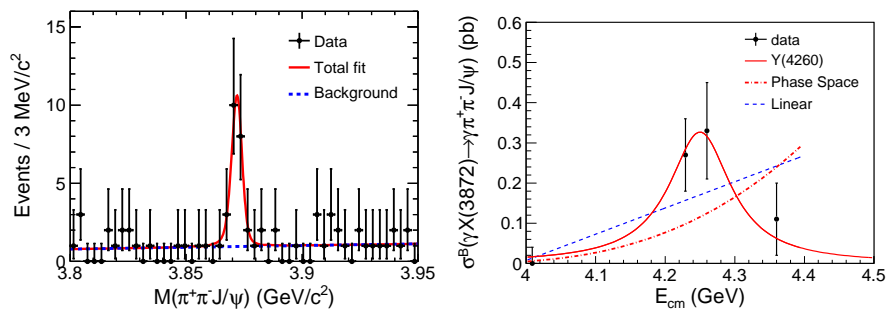

Figure 1: Left: Fit of the $M\left(\pi^{+} \pi^{-} J / \psi\right)$ distribution of a MC simulated histogram convolved with a Gaussian function for signal and a linear background function. Right: The fit to $\sigma^{B}\left(e^{+} e^{-} \rightarrow \gamma X(3872) \rightarrow\right.$ $\left.\gamma \pi^{+} \pi^{-} J / \psi\right)$ with a Y (4260) resonance (red solid curve), a linear continuum (blue dashed curve), or a E1transition phase space term (red dotted-dashed curve). Dots with error bars are data.

\subsection{Observation of $e^{+} e^{-} \rightarrow \pi^{+} \pi^{-} \mathrm{X}(3823)$}

With the data samples at c.m. energies 4.230, 4.260, 4.360, 4.420, and $4.600 \mathrm{GeV}$, BESIII observed the $\mathrm{X}(3823)$ in the process $e^{+} e^{-} \rightarrow \pi^{+} \pi^{-} \mathrm{X}(3823) \rightarrow \pi^{+} \pi^{-} \gamma \chi_{c 1}$ with a statistical significance of $6.2 \sigma$ [9]. The $\pi^{+} \pi^{-}$recoil mass distributions for the event candidates in the $\chi_{c 1}$ and $\chi_{c 2}$ signal regions are shown in Fig $\square$ (left and middle). There is a clear X(3823) signal in the $\gamma \chi_{c 1}$ 
mode, but not in the $\gamma \chi_{c 2}$ mode. The measured mass and width are consistent with these of Belle experiment [एँ] ] and indicate $\mathrm{X}(3823)$ is a good candidate for the $\psi\left(1^{3} D_{2}\right)$ charmonium state [Q]. The cross sections are also measured as shown in Fig D (right) for $e^{+} e^{-} \rightarrow \pi^{+} \pi^{-} \mathrm{X}(3823) \rightarrow \pi^{+} \pi^{-} \gamma \chi_{c 1}$, which can not distinguish between the $\mathrm{Y}(4360)$ decay and $\psi(4415)$ decay hypotheses.
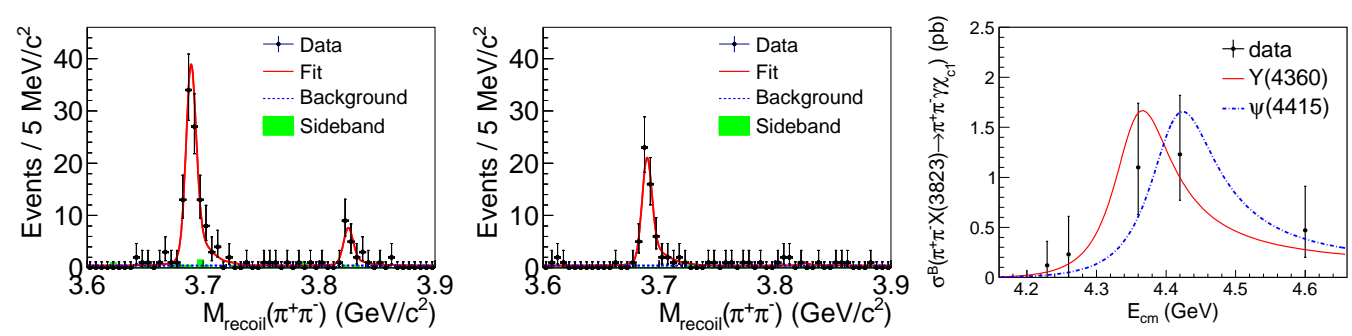

Figure 2: Left and middle: simultaneous fit to the $M_{\text {recoil }}\left(\pi^{+} \pi^{-}\right)$distribution of $\gamma \chi_{c 1}$ events (left) and $\gamma \chi_{c 2}$ events (middle), respectively. Right: Comparison of the energy-dependent cross sections of $e^{+} e^{-} \rightarrow$ $\pi^{+} \pi^{-} \mathrm{X}(3823) \rightarrow \pi^{+} \pi^{-} \gamma \chi_{c 1}$ to the $\mathrm{Y}(4360)$ and $\psi(4415)$ line shapes.

\section{Discovery of the $Z_{c}$ family}

BESIII contributed significantly on the discovery of the $Z_{c}$ family which is summarized in Table س.

Table 1: The summary of the measured mass, width, the decay mode and references for the $Z_{c}$ states observed by BESIII.

\begin{tabular}{|c|c|c|c|c|}
\hline$Z_{c}$ & mass $\left(\mathrm{MeV} / c^{2}\right)$ & width (MeV) & decay mode & references \\
\hline$Z_{c}(3900)^{ \pm}$ & $3899.0 \pm 3.6 \pm 4.9$ & $46 \pm 10 \pm 20$ & $\pi^{ \pm} J / \psi$ & [ए]] \\
\hline$Z_{c}(3900)^{0}$ & $3894.8 \pm 2.3 \pm 2.7$ & $29.6 \pm 8.2 \pm 8.2$ & $\pi^{0} J / \psi$ & [113] \\
\hline$Z_{c}(3885)^{ \pm}$ & $3882.3 \pm 1.1 \pm 1.9$ & $26.5 \pm 1.7 \pm 2.3$ & $\left(D \bar{D}^{*}\right)^{ \pm}$ & [14] [ए5] \\
\hline$Z_{c}(3885)^{0}$ & $3885.7_{-5.7}^{+4.3} \pm 8.4$ & $35_{-12}^{+11} \pm 15$ & $\left(D \bar{D}^{*}\right)^{0}$ & [10] \\
\hline$Z_{c}(4020)^{ \pm}$ & $4022.9 \pm 0.8 \pm 2.7$ & $7.9 \pm 2.7 \pm 2.6$ & $\pi^{ \pm} h_{c}$ & [107] \\
\hline$Z_{c}(4020)^{0}$ & $4023.8 \pm 2.2 \pm 3.8$ & Fixed to 7.9 & $\pi^{0} h_{c}$ & [118] \\
\hline$Z_{c}(4025)^{ \pm}$ & $4026.3 \pm 2.6 \pm 3.7$ & $24.8 \pm 5.6 \pm 7.7$ & $\left(D^{*} \bar{D}^{*}\right)^{ \pm}$ & [एव] \\
\hline$Z_{c}(4025)^{0}$ & $4025.5_{-4.7}^{+2.0} \pm 3.1$ & $23.0 \pm 6.0 \pm 1.0$ & $\left(D^{*} \bar{D}^{*}\right)^{0}$ & [20] \\
\hline
\end{tabular}

\section{1 $Z_{c}(3900)$ and $Z_{c}(3885)$}

A structure, which is refereed to as the $Z_{c}(3900)^{ \pm}$, was first observed in the $\pi^{ \pm} J / \psi$ mass spectrum of the process $e^{+} e^{-} \rightarrow \pi^{+} \pi^{-} J / \psi$ at $4.26 \mathrm{GeV}$ by BESIII [W] and of the process $e^{+} e^{-} \rightarrow$ $\gamma_{I S R} \mathrm{Y}(4260) \rightarrow \gamma_{I S R} \pi^{+} \pi^{-} J / \psi$ by Belle [12]]. A neutral partner, $Z_{c}(3900)^{0}$, was observed in the process $e^{+} e^{-} \rightarrow \pi^{0} \pi^{0} J / \psi$ between the c.m. energy 4.19 and $4.42 \mathrm{GeV}$ at BESIII [[13]. The signals of $Z_{c}(3900)^{ \pm}$and $Z_{c}(3900)^{0}$ are shown in Fig B.

As the mass of $Z_{c}(3900)$ is just above the $D \bar{D}^{*}$ mass threshold, BESIII studied $e^{+} e^{-} \rightarrow$ $\pi^{ \pm}\left(D \bar{D}^{*}\right)^{\mp}$ and observed a structure $Z_{c}(3885)^{ \pm}$in the $\left(D \bar{D}^{*}\right)^{ \pm}$mass spectrum [144, [15]. A neutral partner $Z_{c}(3885)^{0}$ was also observed in the process $e^{+} e \rightarrow\left(D \bar{D}^{*}\right)^{0} \pi^{0}$ [ए6]]. 

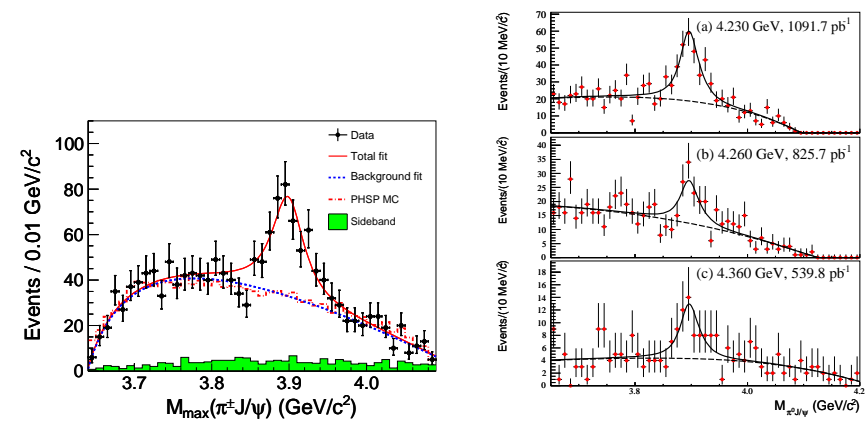

Figure 3: Unbinned maximum likelihood fit to the distribution of the $M_{\max }(\pi J / \psi)$. Points with error bars are data, the curves are the best fit, the dashed histograms are the phase space distributions and the shaded histograms are the non- $\pi^{+} \pi^{-} J / \psi$ background estimated from the normalized $J / \psi$ sidebands.

$3.2 Z_{c}(4020)$ and $Z_{c}(4025)$

A structure $Z_{c}(4020)^{ \pm}$was observed by BESIII in the $\pi^{ \pm} h_{c}$ mass spectrum of the process $e^{+} e^{-} \rightarrow \pi^{+} \pi^{-} h_{c}$ at c.m. energies of 3.90 to $4.42 \mathrm{GeV}$ [ㅁ]]. A neutral partner was also observed in the process $e^{+} e^{-} \rightarrow \pi^{0} \pi^{0} h_{c}$ by BESIII [ए]]]. These signals are shown in Fig. 团.

In the process $e^{+} e^{-} \rightarrow\left(D^{*} \bar{D}^{*}\right)^{ \pm} \pi^{\mp}$, BESIII also observed a structure $Z_{c}(4025)^{ \pm}$near $\left(D^{*} \bar{D}^{*}\right)^{ \pm}$ threshold [ㅁ]. Similarly, a neutral partner $Z_{c}(4025)^{0}$ was observed in the process $e^{+} e^{-} \rightarrow\left(D^{*} \bar{D}^{*}\right)^{0} \pi^{0}$ [20]
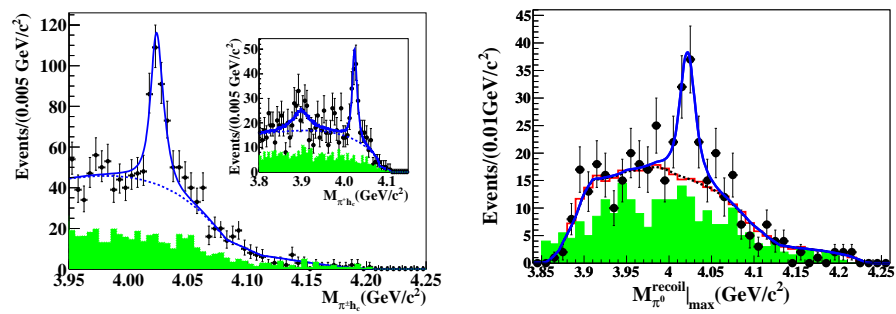

Figure 4: Sum of the simultaneous fits to the $M\left(\pi^{ \pm} h_{c}\right)$ (left) and $M\left(\pi^{0} h_{c}\right)$ (right) distributions at 4.23, 4.26, and $4.36 \mathrm{GeV}$ in the BESIII data; the inset in the left panel shows the sum of the simultaneous fit to the $M_{\pi^{+} h_{c}}$ distributions at 4.23 and $4.26 \mathrm{GeV}$ with $Z_{c}(3900)^{ \pm}$and $Z_{c}(4020)^{ \pm}$. Dots with error bars are data; shaded histograms are normalized sideband background; the solid curves show the total fit, and the dotted curves the backgrounds from the fit.

\section{Measurements of the cross section of $e^{+} e^{-} \rightarrow$ hadron + charmonium}

\subsection{Measurement of $\sigma\left(e^{+} e^{-} \rightarrow \eta\left({ }^{\prime}\right) J / \psi\right)$}

The Born cross section of $e^{+} e^{-} \rightarrow \eta J / \psi$ was measured at energies 3.81 to $4.6 \mathrm{GeV}$ by BESIII [R] [R2] as shown in Fig. [ (left), which are consistent with previous Belle result [[23]. The process $e^{+} e^{-} \rightarrow \eta^{\prime} J / \psi$ was observed for the first time [24] and the measured Born cross section is shown in Fig. [ [ (right) which indicates $\psi(4160) \rightarrow \eta^{\prime} J / \psi$. 

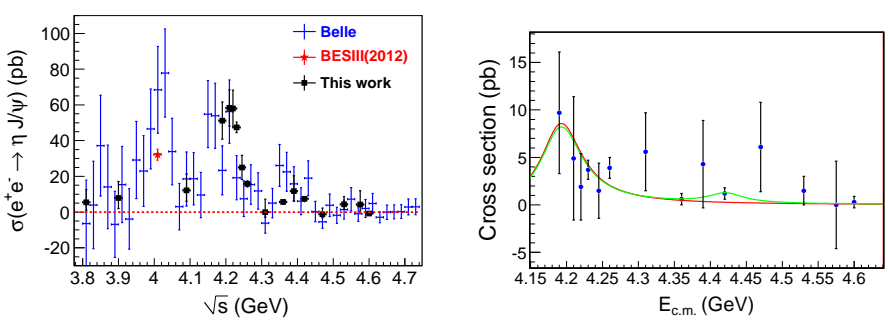

Figure 5: Left: the measured Born cross section of $e^{+} e^{-} \rightarrow \eta J / \psi$ by BESIII (red star dot and black square dots) and by Belle (blue dots). Right: Fit to the Born cross section of $e^{+} e \rightarrow \eta^{\prime} J / \psi$ with a $\psi(4160)$ resonance (red curve), or a combination of $\psi(4160)$ and $\psi(4415)$ resonances (green curve). The uncertainties are statistical only.

4.2 Measurement of $\sigma\left(e^{+} e^{-} \rightarrow \omega \chi_{c J}\right)$

BESIII also observed for the first time the processes $e^{+} e^{-} \rightarrow \omega \chi_{c J}(J=0,1,2)$ [[25] [26], and the measured Born cross sections indicate $\mathrm{Y}(4260) \rightarrow \omega \chi_{c 0}$ and $\psi(4415) \rightarrow \omega \chi_{c 2}$.

\section{Summary}

Using the unique $e^{+} e^{-}$data samples at energies between 3.8 and $4.6 \mathrm{GeV}$, BESIII contributed significantly on the study of the XYZ states. BESIII will continue these studies and will take more data in the energy region.

\section{References}

[1] A. J. Bevan et al. (BaBar and Belle Collaborations), The Physics of the B Factories, Eur. Phys. J. C 74, 3026 (2014).

[2] For instance, a recent review: N. Brambilla et al., Heavy quarkonium: progress, puzzles, and opportunities, Eur. Phys. J. C 71, 1534 (2011).

[3] M. Ablikim et al. (BESIII Collaboration), Design and construction of the BESIII detector, Nucl. Instrum. Methods Phys. Res., Sect. A 614, 345 (2010).

[4] D. M. Asner et al., Physics at BES-III, Int. J. Mod. Phys. A 24, S1 (2009).

[5] M. Ablikim et al. (BESIII Collaboration), Measurement of the center-of-mass energies at BESIII via the di-muon process, Chinese Physics C Vol. 40, No. 6, 063001 (2016).

[6] M. Ablikim et al. (BESIII Collaboration), Precise measurement of the integrated luminosity of the data taken by BESIII at center-of-mass energies between $3.810 \mathrm{GeV}$ and $4.600 \mathrm{GeV}$, Chinese Physics C Vol. 39, No. 9, 093001 (2015).

[7] M. Ablikim et al. (BESIII Collaboration), Observation of $e^{+} e^{-} \rightarrow \gamma X(3872)$ at BESIII, Physical Review Letters 112, 092001 (2014).

[8] K.A. Olive et al. (Particle Data Group), The Review of Particle Physics (2015), Chin. Phys. C, 38, 090001 (2014) and 2015 update.

[9] M. Ablikim et al. (BESIII Collaboration), Observation of the $\psi\left(1^{3} D_{2}\right)$ State in $e^{+} e \rightarrow \pi^{+} \pi^{-} \gamma \chi_{c 1}$ at BESIII, Physical Review Letters 115, 011803 (2015). 
[10] V. Bhardwaj et al. (Belle Collaboration), Evidence of a new narrow resonance decaying to $\chi_{c 1} \gamma$ in $B \rightarrow \chi_{c 1} \gamma K$, Phys. Rev. Lett. 111, no. 3, 032001 (2013).

[11] M. Ablikim et al. (BESIII Collaboration), Observation of a Charged Charmoniumlike Structure in $e^{+} e^{-} \rightarrow \pi^{+} \pi^{-} J / \psi$ at $\sqrt{s}=4.26$ GeV, Phys. Rev. Lett. 110, 252001 (2013).

[12] Z. Q. Liu et al. (Belle Collaboration), Study of $e^{+} e^{-} \rightarrow \pi^{+} \pi^{-} J / \psi$ and Observation of a Charged Charmoniumlike State at Belle, Phys. Rev. Lett. 110, 252002 (2013).

[13] M. Ablikim et al., (BESIII Collaboration), Observation of $Z_{c}(3900)^{0}$ in $e^{+} e \rightarrow \pi^{0} \pi^{0} J / \psi$, Phys. Rev. Lett. 110, 252001 (2013).

[14] M. Ablikim et al., (BESIII Collaboration), Observation of a charged $\left(D \bar{D}^{*}\right)^{ \pm}$mass peak in $e^{+} e \rightarrow \pi D \bar{D}^{*}$ at $\sqrt{s}=4.26 \mathrm{GeV}$, Phys. Rev. Lett. 112, 022001 (2014).

[15] M. Ablikim et al., (BESIII Collaboration), Confirmation of a charged charmoniumlike state $Z_{c}(3885)^{\mp}$ in $e^{+} e \rightarrow \pi^{ \pm}\left(D D^{*}\right)^{\mp}$ with double D tag, Phys. Rev. D 92, 092006 (2015).

[16] M. Ablikim et al., (BESIII Collaboration), Observation of a Neutral Structure near the D $\bar{D}^{*}$ Mass Threshold in $e^{+} e \rightarrow\left(D \bar{D}^{*}\right)^{0} \pi^{0}$ at $\sqrt{(s)}=4.226$ and $4.257 \mathrm{GeV}$, Phys. Rev. Lett. 115, 222002 (2015).

[17] M. Ablikim et al. (BESIII Collaboration), Observation of a charged charmoniumlike structure $Z_{c}(4020)$ and search for the $Z_{c}$ (3900) in $e^{+} e \rightarrow \pi^{+} \pi^{-} h_{c}$, Phys. Rev. Lett. 111, 242001 (2013).

[18] M. Ablikim et al. (BESIII Collaboration), Observation of $e^{+} e \rightarrow \pi^{0} \pi^{0} h_{c}$ and a Neutral Charmoniumlike Structure $Z_{c}(4020)^{0}$, Phys. Rev. Lett. 113, no. 21, 212002 (2014).

[19] M. Ablikim et al. (BESIII Collaboration), Observation of a charged charmoniumlike structure in $e^{+} e \rightarrow\left(D^{*} \bar{D}^{*}\right)^{ \pm} \pi^{\mp}$ at $\sqrt{s}=4.26 \mathrm{GeV}$, Phys. Rev. Lett. 112, 132001 (2014).

[20] M. Ablikim et al. (BESIII Collaboration), Observation of a neutral charmoniumlike state $Z_{c}(4025)^{0}$ in $e^{+} e \rightarrow\left(D^{*} \bar{D}^{*}\right)^{0} \pi^{0}$, Phys. Rev. Lett. 115, 182002 (2015).

[21] M. Ablikim et al. (BESIII Collaboration), Observation of $e^{+} e^{-} \rightarrow \eta J / \psi$ at center-of-mass energy $\sqrt{s}=4.009 \mathrm{GeV}$, Phys. Rev. D 86, 071101(R) (2012).

[22] M. Ablikim et al. (BESIII Collaboration), Measurement of the $e^{+} e^{-} \rightarrow \eta J / \psi$ cross section and search for $e^{+} e^{-} \rightarrow \pi^{0} \mathrm{~J} / \psi$ at center-of-mass energies between 3.810 and $4.600 \mathrm{GeV}$, Phys. Rev. D 91, 112005 (2015).

[23] X. L. Wang et al. (Belle Collaboration), Observation of $\psi(4040)$ and $\psi(4160)$ decay into $\eta J / \psi$, Phys. Rev. D 87, no. 5, 051101 (2013).

[24] M. Ablikim et al. (BESIII Collaboration), Observation of $e^{+} e \rightarrow \eta^{\prime} J / \psi$ at center-of-mass energies between 4.189 and $4.600 \mathrm{GeV}$, arXiv:1605.03256 [hep-ex].

[25] M. Ablikim et al. (BESIII Collaboration), Study of $e^{+} e^{-} \rightarrow \omega \chi_{c J}$ at Center of Mass Energies from 4.21 to $4.42 \mathrm{GeV}$, Phys. Rev. Lett. 114, 092003 (2015).

[26] M. Ablikim et al. (BESIII Collaboration), Observation of $e^{+} e^{-} \rightarrow \omega \chi_{c 1,2}$ near $\sqrt{s}=4.42$ and 4.6 GeV, Phys. Rev. D 93, 011102(R) (2016). 